\title{
On the Line Graph of the Complement Graph for the Ring of Gaussian Integers Modulo $n$
}

\author{
Manal Ghanem ${ }^{1}$, Khalida Nazzal ${ }^{2}$ \\ ${ }^{1}$ Department of Mathematics, Irbid National University, Irbid, Jordan \\ ${ }^{2}$ Department of Mathematics, Palestine Technical University (Kadoorie), Tulkarm, Palestine \\ Email: dr_mghanem@yahoo.com,k.nazzal@ptuk.edu.ps
}

Received October 13, 2011; revised November 2, 2011; accepted November 22, 2011

\begin{abstract}
The line graph for the complement of the zero divisor graph for the ring of Gaussian integers modulo $\mathrm{n}$ is studied. The diameter, the radius and degree of each vertex are determined. Complete characterization of Hamiltonian, Eulerian, planer, regular, locally $H$ and locally connected $L\left(\overline{\Gamma\left(\mathbb{Z}_{n}[i]\right)}\right)$ is given. The chromatic number when $n$ is a power of a prime is computed. Further properties for $L\left(\Gamma\left(\mathbb{Z}_{n}[i]\right)\right)$ and $\overline{\Gamma\left(\mathbb{Z}_{n}[i]\right)}$ are also discussed.
\end{abstract}

Keywords: Complement of a Graph; Chromatic Index; Diameter; Domination Number; Eulerian Graph; Gaussian Integers Modulo $n$; Hamiltonian Graph; Line Graph; Radius; Zero Divisor Graph

\section{Introduction}

The line graph $L(G)$ of a graph $G$ is defined to be the graph whose vertex set constitutes of the edges of $G$, Where two vertices are adjacent if the corresponding edges have a common vertex in $G$. The importance of line graphs stems from the fact that the line graph transforms the adjacency relations on edges to adjacency relations on vertices. For example, the chromatic index of a graph leads to the chromatic number of its line graph. The zero divisor graph of a commutative ring $R$, denoted by $\Gamma(R)$, is defined as the graph whose vertex set is the set of all non-zero zero divisors of $R$ and edge set $E(\Gamma(R))=\{x y: x, y \in R-\{0\}$ and $x y=0\}$. This type of graphs provides an example showing that algebraic methods could be applied to problems about graphs. The set of Gaussian integers, denoted by $\mathbb{Z}[i]$, is defined as the set of complex numbers $a+b i$, where $a, b \in \mathbb{Z}$. If $x$ is a prime Gaussian integer, then $x$ is either

1) $(1+i)$ or $(1-i)$, or

2) $q$ where $q$ is a prime integer and $q \equiv 3(\bmod 4)$, or

3) $a+b i, a-b i$ where $a^{2}+b^{2}=p, p$ is a prime integer and $p \equiv 1(\bmod 4)$.

Throughout this paper, $p$ and $p_{i}$ denote prime integers which are congruent to 1 modulo 4 , while $q$ and and $q_{i}$ denote prime integers which are congruent to 3 modulo 4. All rings in this paper are assumed to be commutative with unity. The zero divisor graph for the ring of Gaussian integers modulo $n$ is studied in [1] and [2], the complement of this graph is discussed in [3]. While the line graph of the zero divisor graph for the ring of Gaussian integers modulo $n$ is investigated in [4]. In this paper it should be kept in mind that

$V\left(\Gamma\left(\mathbb{Z}_{2}[i]\right)\right)=\{1+i\}$, and hence, its line graph is $K_{0}$, $\mathbb{Z}_{q}[i]$ is an integral domain, so $\overline{\Gamma\left(\mathbb{Z}_{q}[i]\right)}=K_{0}$. Further, $\Gamma\left(\mathbb{Z}_{q^{2}}[i]\right)$ is a complete graph whose complement is totally disconnected and thus its line graph is $K_{0}$. While $\Gamma\left(\mathbb{Z}_{p}[i]\right)=K_{p-1, p-1}$, so its complement is disconnected with two components each of which is isomorphic to $K_{p-1}$. Finally, note that the graph $\Gamma\left(\mathbb{Z}_{2 q}[i]\right)$ is bipartite, [1] and $\Gamma\left(\mathbb{Z}_{q_{1} q_{2}}[i]\right)=K_{q_{1}^{2}-1, q_{2}^{2}-1}$.

In this paper, we investigate properties of the graph $L\left(\overline{\Gamma\left(\mathbb{Z}_{n}[i]\right)}\right)$. We find the diameter, the radius of

$L\left(\overline{\Gamma\left(\mathbb{Z}_{n}[i]\right)}\right)$. We determine which $L\left(\overline{\Gamma\left(\mathbb{Z}_{n}[i]\right)}\right)$ is Eulerian, Hamiltonian, regular, locally $H$, locally connected or planer. Furthermore, the chromatic index and the edge domination number of $\Gamma\left(\mathbb{Z}_{n}[i]\right)$ where $n$ is a power of a prime are computed. While the domination number of $\overline{\Gamma\left(\mathbb{Z}_{n}[i]\right)}$ is given. On the other hand, a formula which gives the degree of each vertex in $\Gamma\left(\mathbb{Z}_{n}[i]\right)$ is derived, thus the degree of its complement as well as its line graph could easily be found.

2. When Is $L\left(\overline{\Gamma\left(\mathbb{Z}_{n}[i]\right)}\right)$ Eulerian or

\section{Planner}

If $G$ is a connected graph. Then $G$ is Eulerian if and 
only if every vertex of $G$ has even degree. For a finite ring $R$, the line graph $L(\overline{\Gamma(R)})$ of a connected graph $\overline{\Gamma(R)}$ is Eulerian if and only if all vertices of $\overline{\Gamma(R)}$ have the same parity ( see the proof of Lemma 3.10, [5]). On the other hand, if $G$ has both even and odd vertices, then so is its complement. So, for a connected graph $\overline{\Gamma\left(\mathbb{Z}_{n}[i]\right)}$, the graph $L\left(\overline{\Gamma\left(\mathbb{Z}_{n}[i]\right)}\right)$ is Eulerian if and only if all vertices in $\Gamma\left(\mathbb{Z}_{n}[i]\right)$ are either even or all vertices in $\Gamma\left(\mathbb{Z}_{n}[i]\right)$ are all odd. But $\overline{\Gamma\left(\mathbb{Z}_{n}[i]\right)}$ is connected if $n \neq p, 2^{m}, q^{m}, q_{1} q_{2} \quad[3]$ and $\Gamma\left(\mathbb{Z}_{n}[i]\right)$ is Eulerian if $n=2, p$ or $n$ is a product of distinct odd primes [1]. It is easy to show that all vertices of $\Gamma\left(\mathbb{Z}_{n}[i]\right)$ are odd if and only if $n=q^{2}$. This proves the following theorem.

Theorem 2.1 $L\left(\overline{\Gamma\left(\mathbb{Z}_{n}[i]\right)}\right)$ is Eulerian if and only if $n$ is a product of distinct odd primes.

A planar graph is a graph that can be embedded in the plane, i.e., it can be drawn on the plane in such a way that its edges intersect only at their endpoints.

Next we determine when the graph $L\left(\overline{\Gamma\left(\mathbb{Z}_{n}[i]\right)}\right)$ is planar.

In a graph $G$ the maximum vertex degree and the minimum vertex degree will be denoted by $\Delta(G)$ and $\delta(G)$, respectively.

The following theorem characterizes graphs $G$ whose line graph $L(G)$ is planer.

Theorem 2.2 [6]

A nonempty graph $G$ has a planer line graph $L(G)$ if and only if

1) $G$ is planer.

2) $\Delta(\Gamma(G)) \leq 4$, and

3) if $\operatorname{deg}_{G}(v)=4$, then $v$ is a cut vertex.

The graph $\overline{\Gamma\left(\mathbb{Z}_{n}[i]\right)}$ is planer if and only if $n=2,5$ or $q^{2}$ [3]. For $n=2, q^{2}, L\left(\overline{\Gamma\left(\mathbb{Z}_{n}[i]\right)}\right)=K_{0}$. While for $n=5, \overline{\Gamma\left(\mathbb{Z}_{n}[i]\right)}=K_{4} \cup K_{4}$, this graph is regular of degree 3.

Thus we obtain the following.

Theorem 2.3 The graph $L\left(\overline{\Gamma\left(\mathbb{Z}_{n}[i]\right)}\right)$ is planer if and only is $n=5$.

\section{The Diameter of $L\left(\overline{\Gamma\left(\mathbb{Z}_{n}[i]\right)}\right)$}

For a connected graph $G$, the distance, $d(u, v)$, between two vertices $u$ and $v$ is the minimum of the lengths of all $u-v$ paths of $G$. The eccentricity of a vertex $v$ in $G$ is the maximum distance from $v$ to any vertex in $G$. The diameter of $G, \operatorname{diam}(G)$, is the maximum eccentricity among the vertices of $G$. Since $\overline{\Gamma\left(\mathbb{Z}_{n}[i]\right)}$ is connected if $n \neq p, 2^{m}, q^{m}, q_{1} q_{2}$ and each of $\overline{\Gamma\left(\mathbb{Z}_{p}[i]\right)}$ and $\overline{\Gamma\left(\mathbb{Z}_{q_{1} q_{2}}[i]\right)}$ is the union of two complete graphs, while $\overline{\Gamma\left(\mathbb{Z}_{2^{m}}[i]\right)}$ and
$\overline{\Gamma\left(\mathbb{Z}_{q^{m}}[i]\right)}, m \geq 3$ are the union of a nullgraph and a connected graph [3], we have the following.

Theorem $3.1 L\left(\Gamma\left(\mathbb{Z}_{n}[i]\right)\right)$ is connected if and only if $n \neq 2, p, q^{2}, q_{1} q_{2}$.

Theorem 3.2 If $n=2^{m}, m \geq 2$ or $n=q^{m}, m \geq 3$, then $\operatorname{diam}\left(L\left(\overline{\Gamma\left(\mathbb{Z}_{n}[i]\right)}\right)\right)=2$.

Proof. 1) Assume that $n=2^{m}, m \geq 2$ and

$$
\left[x=x_{1}+x_{2} i, y=y_{1}+y_{2} i\right],\left[z=z_{1}+z_{2} i, w=w_{1}+w_{2} i\right]
$$

are two nonadjacent vertices in $V\left(L\left(\overline{\Gamma\left(\mathbb{Z}_{n}[i]\right)}\right)\right)$. Since for every $a+b i \in \mathbb{Z}_{2^{m}}[i], a$ and $b$ are both even or odd [1], we have three cases:

Case I: for $i=1,2, x_{i}, y_{i}, z_{i}$ and $w_{i}$ are odd. Then we have the path $[x, y]---[x, z]---[z, w]$.

Case II: for $i=1,2, x_{i}$ or $y_{i}$ is odd(even) and $z_{i}$ or $w_{i}$ is even (odd). Assume that $x_{1}, x_{2}$ are even and $z_{1}, z_{2}$ are odd. Then we have the path

$[x, y]---[x, z]---[z, w]$.

Case III: for $i=1,2, x_{i}, y_{i}, z_{i}$ and $w_{i}$ are even. Then $[x, y]=\left[\alpha_{1} 2^{t_{1}}+\beta_{1} 2^{s_{1}} i, \alpha_{2} 2^{t_{2}}+\beta_{2} 2^{s_{2}} i\right]$ and $[z, w]=\left[\alpha_{3} 2^{t_{3}}+\beta_{3} 2^{s_{3}} i, \alpha_{4} 2^{t_{4}}+\beta_{4} 2^{s_{4}} i\right]$ where $\alpha_{i}, \beta_{i}$ are odd and $1 \leq t_{i}, s_{i} \leq m$ for $1 \leq i \leq 4$. If $t_{1}, s_{1}, t_{2}$, or $s_{2}<\left\lfloor\frac{m}{2}\right\rfloor$, say $t_{1}$, then $t_{3}, s_{3}, t_{4}$ or $s_{4}<m-t_{1}$, say $t_{3}$. So, we have the path $[x, y]---[x, z]---[z, w]$. Now suppose that $m$ is odd. Then

a) If $t_{i}=s_{i}=\frac{m-1}{2}, \alpha_{i} \neq \beta_{i}$, for $i=1$ or 2 , say for $i=1$, then $t_{3}, s_{3}, t_{4}$ or $s_{4}<m-t_{1}$, say $t_{3}$. Hence, we have the path $[x, y]---[x, z]---[z, w]$.

b) If $t_{i}$ or $s_{i}=\frac{m-1}{2}$ and $t_{i} \neq s_{i}$, for $i=1$ or 2 , say for $i=1$, then we have a path

$[x, y]---[x, z]---[z, w]$ or

$[x, y]---[x, w]---[z, w]$.

c) If $t_{i}=s_{i}=\frac{m-1}{2}, \alpha_{i}=\beta_{i}$, for $i=1$ or 2 , say for $i=1$, then $t_{2}=s_{2}=\frac{m-1}{2}$ implies that $\alpha_{2} \neq \beta_{2}$. Otherwise $t_{2}$ or $s_{2} \leq \frac{m-1}{2}$. Then we have a path

$[x, y]---[y, z]---[z, w]$ or $[x, y]---[y, w]---[z, w]$.

2) Assume that $n=q^{m}, m \geq 3$ and

$$
\begin{aligned}
& {\left[\alpha_{1} q^{t_{1}}+\beta_{1} q^{s_{1}} i, \alpha_{2} q^{t_{2}}+\beta_{2} q^{s_{2}} i\right],} \\
& {\left[\alpha_{3} q^{t_{3}}+\beta_{3} q^{s_{3}} i, \alpha_{4} q^{t_{4}}+\beta_{4} q^{s_{4}} i\right] \in V\left(L\left(\overline{\Gamma\left(\mathbb{Z}_{n}[i]\right)}\right)\right)}
\end{aligned}
$$


Then $t_{1}, s_{1}, t_{2}$ or $s_{2}<\left\lceil\frac{m}{2}\right\rceil$, say $t_{1}$. Hence $t_{3}, s_{3}, t_{4}$ or $s_{4}<m-t_{1}$, say $t_{3}$. Then we have the path

$$
\begin{aligned}
& {\left[\alpha_{1} q^{t_{1}}+\beta_{1} q^{s_{1}} i, \alpha_{2} q^{t_{2}}+\beta_{2} q^{s_{2}} i\right]---} \\
& {\left[\alpha_{1} q^{t_{1}}+\beta_{1} q^{s_{1}} i, \alpha_{3} q^{t_{3}}+\beta_{3} q^{s_{3}} i\right]---.} \\
& {\left[\alpha_{3} q^{t_{3}}+\beta_{3} q^{s_{3}} i, \alpha_{4} q^{t_{4}}+\beta_{4} q^{s_{4}} i\right]}
\end{aligned}
$$

Theorem 3.3 Let $R$ be a ring that is a product of two rings $R_{1}$ and $R_{2}$ with at least one of them is not ID with more than one regular element and the other has more than two regular elements. Then $\operatorname{diam}(L(\overline{\Gamma(R)}))=3$.

Proof. Suppose that $R=R_{1} \times R_{2}$ and $R_{1}$ is not ID, $\left|\operatorname{reg}\left(R_{1}\right)\right| \geq 2$ and $\left|\operatorname{reg}\left(R_{2}\right)\right| \geq 3$. Let $z_{1} \in V\left(\Gamma\left(R_{1}\right)\right)$ and $u_{2} \in \operatorname{reg}\left(R_{2}\right)-\{1\}$. Clearly,

$d\left(\left[(1,0),\left(z_{1}, 0\right)\right],\left[(0,1),\left(0, u_{2}\right)\right]\right)=3$ in $L(\overline{\Gamma(R)})$. So, $\operatorname{diam}(L(\overline{\Gamma(R)})) \geq 3$. Now, let

$$
\left[\left(a_{1}, a_{2}\right),\left(b_{1}, b_{2}\right)\right],\left[\left(c_{1}, c_{2}\right),\left(d_{1}, d_{2}\right)\right] \in V(L(\overline{\Gamma(R)})),
$$

then $a_{1} b_{1} \neq 0$ or $a_{2} b_{2} \neq 0$ and $c_{1} d_{1} \neq 0$ or $c_{2} d_{2} \neq 0$. So, we have three cases:

Case I: $a_{1} b_{1} \neq 0$ and $c_{1} d_{1} \neq 0$. Then $a_{1}, c_{1} \in \operatorname{reg}\left(R_{1}\right)$ implies that

$$
\left[\left(z_{1}, 0\right),\left(a_{1}, a_{2}\right)\right]\left[\left(z_{1}, 0\right),\left(c_{1}, c_{2}\right)\right] \in E(L(\overline{\Gamma(R)})) .
$$

And $a_{1}$ or $c_{1} \in Z\left(R_{1}\right)$, say $a_{1}$ implies that

$$
\left[\left(u_{1}, 0\right),\left(a_{1}, a_{2}\right)\right]\left[\left(u_{1}, 0\right),\left(c_{1}, c_{2}\right)\right] \in E(L(\overline{\Gamma(R)}))
$$

where $u_{1} \in \operatorname{reg}\left(R_{1}\right)-\left\{c_{1}\right\}$.

Case II: $a_{2} b_{2} \neq 0$ and $c_{2} d_{2} \neq 0$. Then there exists $v_{2} \in \operatorname{reg}\left(R_{2}\right)-\left\{a_{2}, c_{2}\right\}$ and hence

$$
\left[\left(a_{1}, a_{2}\right),\left(0, v_{2}\right)\right]\left[\left(c_{1}, c_{2}\right),\left(0, v_{2}\right)\right] \in E(L(\overline{\Gamma(R)})) .
$$

Case III: $a_{1} b_{1} \neq 0$ and $c_{2} d_{2} \neq 0$ or $a_{2} b_{2} \neq 0$ and $c_{1} d_{1} \neq 0$. Let $a_{1} b_{1} \neq 0$ and $c_{2} d_{2} \neq 0$. Then $a_{1} \in \operatorname{reg}\left(R_{1}\right)$ implies that $\left[\left(a_{1}, a_{2}\right),\left(z_{1}, c_{2}\right)\right] \in V(L(\overline{\Gamma(R)}))$ and $\left(z_{1}, c_{2}\right)=\left(d_{1}, d_{2}\right)$ or $\left[\left(d_{1}, d_{2}\right),\left(z_{1}, c_{2}\right)\right] \in V(L(\overline{\Gamma(R)}))$. And if $a_{1} \in Z\left(R_{1}\right)$, then $\left(a_{1}, c_{2}\right)=\left(b_{1}, b_{2}\right)$ or $\left[\left(a_{1}, c_{2}\right),\left(b_{1}, b_{2}\right)\right] \in V(L(\overline{\Gamma(R)}))$ and $\left(a_{1}, c_{2}\right)=\left(d_{1}, d_{2}\right)$ or $\left[\left(a_{1}, c_{2}\right),\left(d_{1}, d_{2}\right)\right] \in V(L(\overline{\Gamma(R)}))$.

For $n=p^{m}, \quad \mathbb{Z}_{n}[i] \cong \mathbb{Z}_{p^{m}} \times \mathbb{Z}_{p^{m}}[7]$ and for $n=n_{1} n_{2}$ with $\operatorname{g.c.d}\left(n_{1}, n_{2}^{p^{m}}\right)=1,{ }^{p^{m}} \mathbb{Z}_{n}[i] \cong \mathbb{Z}_{n_{1}}[i] \times \mathbb{Z}_{n_{2}}[i]$. Moreover $\left|\operatorname{reg}\left(\mathbb{Z}_{2}[i]\right)\right|=2$ and $\left|\operatorname{reg}\left(\mathbb{Z}_{m}[i]\right)\right| \geq 3$ for $m \neq 2$. An immediate consequence of Theorem 3.3 is the following.

Theorem 3.4 Let $n=p^{m}, m \geq 2$ or $n$ is a composite such that $n \neq q_{1} q_{2}$. Then

$$
\operatorname{diam}\left(L\left(\overline{\Gamma\left(\mathbb{Z}_{n}[i]\right)}\right)\right)=3 .
$$

\section{The Radius and the Girth of the Graph $L\left(\overline{\Gamma\left(\mathbb{Z}_{n}[\mathbf{i}]\right)}\right)$}

For a connected graph $G$, the radius of $G, \operatorname{rad}(G)$, is the minimum eccentricity among the vertices of $G$. So, $\operatorname{rad}(G) \leq \operatorname{diam}(G)$. Since for any

$[a, b] \in V\left(L\left(\overline{\Gamma\left(\mathbb{Z}_{n}[i]\right)}\right)\right),[a, b]$ and $[a i, b i]$ are non adjacent, $\operatorname{rad}\left(L\left(\overline{\Gamma\left(\mathbb{Z}_{n}[i]\right)}\right)\right)>1$. Using Theorem 3.2 gives for $n=2^{m}, m \geq 2$ or $n=q^{m}, m \geq 3$, $\operatorname{rad}\left(L\left(\overline{\Gamma\left(\mathbb{Z}_{n}[i]\right)}\right)\right)=2$.

Theorem 4.1 If $n=p^{m}, m \geq 2$ or $n=t^{m} s$ where $m \geq 1, t$ is prime integer, g.c.d $(t, s)=1$ and $n \neq q_{1} q_{2}$, then $\operatorname{rad}\left(L\left(\overline{\Gamma\left(\mathbb{Z}_{n}[i]\right)}\right)\right)=2$.

Proof. Since $\operatorname{rad}\left(L\left(\overline{\Gamma\left(\mathbb{Z}_{n}[i]\right)}\right)\right)>1$ to show that $\operatorname{rad}\left(L\left(\overline{\Gamma\left(\mathbb{Z}_{n}[i]\right)}\right)\right)=2$ it is enough to find a vertex $v \in V\left(L\left(\overline{\Gamma\left(\mathbb{Z}_{n}[i]\right)}\right)\right)$ with eccentricity 2 . If $n=p^{m}, p=a^{2}+b^{2}, m \geq 2$, then $d([a+b i, a-b i],[x, y]) \leq 2$ for every $[x, y] \in V\left(L\left(\overline{\Gamma\left(\mathbb{Z}_{n}[i]\right)}\right)\right)$. So $\operatorname{rad}\left(L\left(\overline{\Gamma\left(\mathbb{Z}_{p^{m}}[i]\right)}\right)\right)=2$.

Now, assume that $n=t^{m} s, m \geq 1$ and

$$
[(x, y),(w, z)] \in V\left(L\left(\overline{\Gamma\left(\mathbb{Z}_{n}[i]\right)}\right)\right) .
$$

Then we have four cases:

Case I: $t=2$. Then

$$
d([(1+i, 1),(1,0)],[(x, y),(w, z)]) \leq 2 .
$$

Case II: $t=p$. Then

$$
d([(a+b i, 1),(a-b i, 1)],[(x, y),(w, z)]) \leq 2 .
$$

Case III: $t=q_{1}$ and $m=1$. Then $s \neq q_{2}$ and hence there exists $a \in V\left(\Gamma\left(\mathbb{Z}_{s}[i]\right)\right)$. So,

$$
d([(0,1),(1, a)],[(x, y),(w, z)]) \leq 2 .
$$

Case IV: $t=q, m \geq 2$. Then

$$
d([(q, 1),(1,0)],[(x, y),(w, z)]) \leq 2 .
$$

Theorem $4.2 \operatorname{rad}\left(L\left(\overline{\Gamma\left(\mathbb{Z}_{n}[i]\right)}\right)\right)=2$ if and only if $n \neq 2, p, q, q^{2}$ or $q_{1} q_{2}$. 
Vising [8], proved that for a connected simple graph $G$ with $n$-vertices and radius 2, the upper bound of the number of edges of $G$ is $\frac{n(n-2)}{2}$. Then Golberg [9] proved that the lower bound of numbers of edges of a simple connected graph $G$ with radius 2 is $\frac{3(n-1)}{4}$. So we can conclude the following.

Theorem 4.3 For $n \neq 2, p, q, q^{2}$ or $q_{1} q_{2}$, $\left|L\left(\overline{\Gamma\left(\mathbb{Z}_{n}[i]\right)}\right)\right|=t$ implies that

$$
\frac{3(t-1)}{4} \leq\left|E\left(L\left(\overline{\Gamma\left(\mathbb{Z}_{n}[i]\right)}\right)\right)\right| \leq \frac{t(t-2)}{2} .
$$

The girth of a graph $G, g(G)$ is the length of a shortest cycle contained in the graph. If the graph does not contain any cycles (i.e.. it's an acyclic graph), its girth is defined to be infinity. If $a, b, c, a$ is a cycle of length three in $G$. Then $[a, b],[b, c],[c, a],[a, b]$ is a cycle of length 3 in $L(G)$. So, $g(L(G))=3$ whenever $g(G)=3$. In [3] it is proved that the girth of $\overline{\Gamma\left(\mathbb{Z}_{n}[i]\right)}$ equals 3 for $n \neq 2, q, q^{2}$. So, we have the following.

Theorem 4.4 For $n \neq 2, q, q^{2}, g\left(L\left(\overline{\Gamma\left(\mathbb{Z}_{n}[i]\right)}\right)\right)=3$.

\section{The Locally Connected Property of the Graphs $\overline{\Gamma\left(\mathbb{Z}_{n}[i]\right)}$ and $L\left(\overline{\Gamma\left(\mathbb{Z}_{n}[i]\right)}\right)$}

We say that a vertex $v$ is locally connected if the neighborhood of $v, N(v)$, is connected; and $G$ is locally connected if every vertex of $G$ is locally connected.

Theorem 5.1 If $R=R_{1} \times R_{2}, \quad\left|\operatorname{reg}\left(R_{i}\right)\right| \geq 2$ for $i=1,2$ and either $R_{1}$ or $R_{2}$ is not ID, then $\overline{\Gamma(R)}$ is locally connected.

Proof. Suppose that $R_{1}$ is not ID and $(x, y) \in V(\overline{\Gamma(R)})$. Then we have two cases:

Case I: $x=0$ or $y=0$. If $x=0$, then there exists $z_{1} \in V\left(\Gamma\left(R_{1}\right)\right)$. So $\left(z_{1}, 1\right)(a, b) \in E \overline{(\Gamma(R))}$ for all $(a, b) \in N((x, y))$. And if $y=0$, then there exists $u_{1} \in \operatorname{reg}\left(R_{1}\right)-\{x\}$ such that $\left(u_{1}, 0\right) \in N((x, y))$. Therefore, $\left(u_{1}, 0\right)(a, b) \in E(\overline{\Gamma(R)})$ for every $(a, b) \in N((x, y))$. So $N((x, y))$ is connected.

Case II: $x \neq 0$ and $y \neq 0$. Then there exist $v_{1} \in \operatorname{reg}\left(R_{1}\right)-\{x\}, \quad v_{2} \in \operatorname{reg}\left(R_{2}\right)-\{y\}$ and $z_{1} \in V\left(\Gamma\left(R_{1}\right)\right)$ such that $\left(v_{1}, 0\right),\left(z_{1}, v_{2}\right)$ and $\left(0, v_{2}\right) \in N((x, y))$. Moreover,

$\left(v_{1}, 0\right)\left(z_{1}, v_{2}\right),\left(0, v_{2}\right)\left(z_{1}, v_{2}\right) \in E(\overline{\Gamma(R)})$. And for every $(t, s) \in Z(R), \quad\left(v_{1}, 0\right)(t, s)$ or $\left(0, v_{2}\right)(t, s) \in E(\overline{\Gamma(R)})$. So $N((x, y))$ is connected.

Theorem 5.2 If $R=R_{1} \times R_{2}, \quad\left|\operatorname{reg}\left(R_{i}\right)\right| \geq 2$ for $i=1,2$ and either $R_{1}$ or $R_{2}$ is not ID, then $L\left(\frac{\Gamma(R)}{\Gamma(s)}\right.$ is locally connected.

Proof. Suppose that $R_{1}$ is not ID, $z_{1} \in V\left(\Gamma\left(R_{1}\right)\right)$ and $[(x, y),(z, w)] \in V(L(\overline{\Gamma(R)}))$, then we have three cases:

Case I: $x=z=0$. Then

$$
\left[\left(z_{1}, 1\right),(x, y)\right]\left[\left(z_{1}, 1\right),(z, w)\right] \in E(L(\overline{\Gamma(R)})) .
$$

Case II: $y=w=0$. If $x, z \in \operatorname{reg}\left(R_{1}\right)$, then

$$
\left[\left(z_{1}, 1\right),(x, y)\right]\left[\left(z_{1}, 1\right),(z, w)\right] \in E(L(\overline{\Gamma(R)})) .
$$

Otherwise there exists $u_{1} \in \operatorname{reg}\left(R_{1}\right)-\{x, z\}$. So,

$$
\left[\left(u_{1}, 0\right),(x, y)\right]\left[\left(u_{1}, 0\right),(z, w)\right] \in E(L(\overline{\Gamma(R)})) .
$$

Case III: $x, y \neq 0$ or $z, w \neq 0$. Assume that $x y \neq 0$, then $z \neq 0$ implies that there exists $u_{1} \in \operatorname{reg}\left(R_{1}\right)-\{x\}$ satisfies

$$
\left[\left(u_{1}, 0\right),(x, y)\right]\left[\left(u_{1}, 0\right),(z, w)\right] \in E(L(\overline{\Gamma(R)})) .
$$

While $w \neq 0$ implies that that there exists $v_{2} \in \operatorname{reg}\left(R_{2}\right)-\{w\}$ satisfies

$$
\left[\left(0, v_{2}\right),(x, y)\right]\left[\left(0, v_{2}\right),(z, w)\right] \in E(L(\overline{\Gamma(R)})) .
$$

From Theorem 5.1 and Theorem 5.2 we conclude the following.

Theorem 5.3 If $n=p^{m}, m \geq 1$ or $n$ is a composite integer such that $n \neq q_{1} q_{2}$, then both $\overline{\Gamma\left(\mathbb{Z}_{n}[i]\right)}$ and $L\left(\overline{\Gamma\left(\mathbb{Z}_{n}[i]\right)}\right)$ are locally connected.

\section{When Is $L\left(\overline{\Gamma\left(\mathbb{Z}_{n}[i]\right)}\right)$ Hamiltonian?}

A Hamiltonian cycle is a cycle that visits each vertex exactly once (except the vertex which is both the start and end, and so is visited twice). A graph that contains a Hamiltonian cycle is called a Hamiltonian graph. The line graph of a graph $G$ with more than 4 vertices and diameter 2 is Hamiltonian [10]. But $\overline{\Gamma\left(\mathbb{Z}_{2^{m}}[i]\right)}, m \geq 2$ is disconnected with one isolated vertex $\left\{2^{m-1}+2^{m-1} i\right\}$ and the other component, call this component $H$, with diameter 2 [3]. So, $L\left(\overline{\Gamma\left(\mathbb{Z}_{2^{m}}[i]\right)}\right) \cong L(H)$. Similarly, $\overline{\Gamma\left(\mathbb{Z}_{q^{m}}[i]\right)}$ has a connected subgraph $H$ with diameter 2 and $L\left(\overline{\Gamma\left(\mathbb{Z}_{q^{m}}[i]\right)}\right) \cong L(H)$. Hence, the following result is obtained.

Theorem 6.1 If $n=2^{m}, m \geq 2$ or $n=q^{m}, m \geq 3$, then $L\left(\overline{\Gamma\left(\mathbb{Z}_{n}[i]\right)}\right)$ is Hamiltonian.

Oberly and Sumner [11] proved that every connected, locally connected claw free graph (i.e. it does not contain 
a complete bipartite graph $K_{1,3}$ ) is hamiltonian. Since the line graph is claw free, using Theorem 5.3, we get the following.

Theorem 6.2 If $n=p^{m}, m \geq 2$ or $n$ is a composite integer such that $n \neq q_{1} q_{2}$, then $L\left(\overline{\Gamma\left(\mathbb{Z}_{n}[i]\right)}\right)$ is hamiltonian.

\section{The Chromatic Number of the Graph$$
L\left(\overline{\Gamma\left(\mathbb{Z}_{n}[\boldsymbol{i}]\right)}\right)
$$

The edge coloring of a graph $G$ is an assignment of colors to the edges of the graph so that no two adjacent edges have the same color. The minimum required number of colors for the edges of a given graph is called the chromatic index of the graph denoted by $\chi^{\prime}(G)$.

Lemma 7.1 [12]

If $G$ has order $2 s$ and $\Delta(G)=2 s-1$, then $\chi^{\prime}(G)=\Delta(G)$.

Theorem 7.2 If $n=2^{m}, m \geq 2$, then

$$
\chi^{\prime}\left(\overline{\Gamma\left(\mathbb{Z}_{n}[i]\right)}\right)=2^{2 m-1}-3 \text {. }
$$

Proof. Note that in $\overline{\Gamma\left(\mathbb{Z}_{2^{m}}[i]\right)}$, the induced subgraph,

$H$, with $V(H)=V\left(\overline{\Gamma\left(\mathbb{Z}_{2^{m}}[i]\right)}\right)-\left\{2^{m-1}+2^{m-1} i\right\}$ is connected, $|V(H)|=2^{2 m-1}-2,[1]$ and $\chi^{\prime}(H)=\chi^{\prime}\left(\overline{\Gamma\left(\mathbb{Z}_{2^{m}}[i]\right)}\right)$. Since the vertex $1+i$ is adjacent to all other vertices in $H$, we have $\Delta(H)=\operatorname{deg}(1+i)=2^{2 m-1}-3$. Using Lemma 6.1,

$$
\chi^{\prime}\left(\overline{\Gamma\left(\mathbb{Z}_{2^{m}}[i]\right)}\right)=2^{2 m-1}-3 .
$$

Since $\overline{\Gamma\left(\mathbb{Z}_{q}[i]\right)}$ is empty graph and $\overline{\Gamma\left(\mathbb{Z}_{q^{2}}[i]\right)}=\left(q^{2}-1\right) K_{1}$ is edgeless with $q^{2}-1$ vertices, we consider the case $\overline{\Gamma\left(\mathbb{Z}_{q^{m}}[i]\right)}, q \geq 3$.

Theorem 7.3 If $n=q^{m}, m \geq 3$, then

$$
\chi^{\prime}\left(\overline{\Gamma\left(\mathbb{Z}_{n}[i]\right)}\right)=q^{2 m-2}-q^{2}-1
$$

Proof. Let $A=\left\{\alpha q^{m-1}+\beta q^{m-1} i: \alpha, \beta \in \mathbb{Z}_{q}\right\}-\{0\}$.

Then $A$ is the set of all isolated vertices in $\overline{\Gamma\left(\mathbb{Z}_{q^{m}}[i]\right)}$. So the induced subgraph, $H$, with the vertices $V(H)=V\left(\overline{\Gamma\left(\mathbb{Z}_{q^{m}}[i]\right)}\right)-A$ is a connected graph,

$|V(H)|=q^{2 m-2}-q^{2}$. Clearly the vertex $q$ is adjacent to all other vertices in $H$ and hence, $\operatorname{deg}(q)=q^{2 m-2}-q^{2}-1$. Using Lemma 7.1,

$$
\chi^{\prime}\left(\overline{\Gamma\left(\mathbb{Z}_{q^{m}}[i]\right)}\right)=q^{2 m-2}-q^{2}-1 .
$$

Finally we find the chromatic index of $\overline{\Gamma\left(\mathbb{Z}_{p^{m}}[i]\right)}, m \geq 2$.

A subset $D$ of the vertex set $V(G)$ is said to be independent if no two vertices in this set are adjacent. A clique of a graph is a maximal complete subgraph. A graph $G$ is said to be split if it's vertex set can be partitioned into two subsets $A$ and $B$ such that $A$ induces a clique and $B$ is independent in $G$.

Lemma 7.4 [13] Let $G$ be a split graph. If $\Delta(G)$ is odd, then $\chi^{\prime}(G)=\Delta(G)$.

Theorem 7.5 If $n=p^{2}$, then

$$
\chi^{\prime}\left(\overline{\Gamma\left(\mathbb{Z}_{n}[i]\right)}\right)=2 p^{3}-p^{2}-p-1 .
$$

Proof. Since $\mathbb{Z}_{p^{2}}[i] \cong \mathbb{Z}_{p^{2}} \times \mathbb{Z}_{p^{2}}$, it is enough to find $\chi^{\prime}\left(\mathbb{Z}_{p^{2}} \times \mathbb{Z}_{p^{2}}\right)$. First, we'll show that $\overline{\Gamma\left(\mathbb{Z}_{p^{2}} \times \mathbb{Z}_{p^{2}}\right)}$ is a split graph. Let

$$
\begin{aligned}
A= & \left(\left\{(u, \beta p): u \in U\left(\mathbb{Z}_{p^{2}}\right) \text { and } \beta \in \mathbb{Z}_{p}\right\}\right\} \\
& \left.\cup\left\{(\alpha p, v): v \in U\left(\mathbb{Z}_{p^{2}}\right) \text { and } \alpha \in \mathbb{Z}_{p}\right\}\right), \\
B= & \left\{(\alpha p, \beta p): \alpha \text { and } \beta \in \mathbb{Z}_{p}\right\}-\{(0,0)\} .
\end{aligned}
$$

Clearly, $V\left(\overline{\mathbb{Z}_{p^{2}} \times \mathbb{Z}_{p^{2}}}\right)=A \cup B, A$ induces a clique and $B$ is independent. Therefore, $\overline{\Gamma\left(\mathbb{Z}_{p^{2}} \times \mathbb{Z}_{p^{2}}\right)}$ is a split graph. Moreover,

$$
\begin{aligned}
& \Delta\left(\overline{\Gamma\left(\mathbb{Z}_{p^{2}} \times \mathbb{Z}_{p^{2}}\right)}\right)=\operatorname{deg}(1, p) \\
& =\left|\overline{\Gamma\left(\mathbb{Z}_{p^{2}}[i]\right)}\right|-\left|\left\{(0, \beta p): \beta \in \mathbb{Z}_{p}-\{0\}\right\} \cup\{(1, p)\}\right| \\
& =2 p^{3}-p^{2}-p-1
\end{aligned}
$$

is odd. From Lemma 7.4,

$$
\chi^{\prime}\left(\overline{\Gamma\left(\mathbb{Z}_{p^{m}}[i]\right)}\right)=2 p^{3}-p^{2}-p-1 .
$$

A graph $G$ is said to be critical if $G$ is connected and $\chi^{\prime}(G)=\Delta(G)+1$ and for every edge $e$ of $G$, we have $\chi^{\prime}(G \backslash\{e\})<\chi^{\prime}(G)$. The well-known Vizing's theorem states that for a simple graph $G$, $\chi^{\prime}(G)=\Delta(G)$ or $\Delta(G)+1$.

\section{Lemma 7.6 [14]}

If $G$ is a critical graph, then $G$ has at least $\Delta(G)-\delta(G)+2$ of vertices of maximum degree.

Therefore, if $G$ is a simple graph such that for every vertex $v$ of maximum degree there exists an edge $v u$ such that $\Delta(G)-\operatorname{deg}(u)+2$ is more than the number 
of vertices with maximum degree in $G$, we have $\chi^{\prime}(G)=\Delta(G)[13]$.

Theorem 7.7 If $n=p^{m}, m \geq 3$, then

$$
\chi^{\prime}\left(\overline{\Gamma\left(\mathbb{Z}_{n}[i]\right)}\right)=2 p^{2 m-1}-p^{2 m-2}-p-1 .
$$

Proof. Let $\alpha, \beta \in V\left(\Gamma\left(\mathbb{Z}_{p}\right)\right)$ and $u, v \in U\left(\mathbb{Z}_{p^{m}}\right)$. Then the vertices of $\overline{\Gamma\left(\mathbb{Z}_{p^{m}} \times \mathbb{Z}_{p^{m}}\right)}$ with maximum degree have the form $(\alpha p, v)$ or $(u, \beta p)$ where $\alpha \neq 0$ and $\beta \neq 0$ and

$$
\begin{aligned}
N((\alpha p, v)) & =V\left(\overline{\Gamma\left(\mathbb{Z}_{p^{m}} \times \mathbb{Z}_{p^{m}}\right)}\right) \\
& -\left(\left\{\left(\beta p^{m-1}, 0\right): \beta \neq 0\right\} \cup\{(\alpha p, v)\}\right)
\end{aligned}
$$

and

$$
\begin{aligned}
N((u, \beta p)) & =V\left(\overline{\Gamma\left(\mathbb{Z}_{p^{m}} \times \mathbb{Z}_{p^{m}}\right)}\right) \\
& -\left(\left\{\left(0, \alpha p^{m-1}\right): \alpha \neq 0\right\} \cup\{(u, \beta p)\}\right) .
\end{aligned}
$$

So, $\Delta\left(\overline{\Gamma\left(\mathbb{Z}_{p^{m}} \times \mathbb{Z}_{p^{m}}\right)}\right)=2 p^{2 m-1}-p^{2 m-2}-p-1$. And the vertices of $\overline{\Gamma\left(\mathbb{Z}_{p^{m}} \times \mathbb{Z}_{p^{m}}\right)}$ with minimum degree have the form $\left(\alpha p^{m-1}, 0\right)$ or $\left(0, \beta p^{m-1}\right)$ where

$$
\begin{aligned}
& N\left(\left(\alpha p^{m-1}, 0\right)\right)=\left\{\left(u, \alpha p^{i}\right): i \geq 1\right\} \text { and } \\
& N\left(\left(0, \beta p^{m-1}\right)\right)=\left\{\left(\alpha p^{i}, v\right): i \geq 1\right\} \text {. So } \\
& \delta\left(\overline{\left(\Gamma\left(\mathbb{Z}_{p^{m}}[i]\right)\right)}\right)=\left(p^{m}-p^{m-1}\right)(m p-m-p+2) .
\end{aligned}
$$

Therefore,

$$
\begin{aligned}
& \Delta\left(\overline{\Gamma\left(\mathbb{Z}_{p^{m}}[i]\right)}\right)-\delta\left(\overline{\Gamma\left(\mathbb{Z}_{p^{m}} \times \mathbb{Z}_{p^{m}}\right)}\right)+2 \\
& =\left(p^{m}-p^{m-1}\right)\left(p^{m-1}-m p+m+p-2\right)+p^{2 m-1}-p+1 . \\
& >2\left(p^{m}-p^{m-1}\right)(p-1)
\end{aligned}
$$

But the graph $\overline{\Gamma\left(\mathbb{Z}_{p^{m}} \times \mathbb{Z}_{p^{m}}\right)}$ has only $2\left(p^{m}-p^{m-1}\right)(p-1)$ vertices of maximum degree. So,

$$
\chi^{\prime}\left(\overline{\Gamma\left(\mathbb{Z}_{p^{m}} \times \mathbb{Z}_{p^{m}}\right)}\right)=2 p^{2 m-1}-p^{2 m-2}-p-1 .
$$

Since $\mathbb{Z}_{{ }^{m}}[i] \cong \mathbb{Z}_{{ }^{m}} \times \mathbb{Z}_{{ }^{m}}$, the result holds.

Since the edge coloring of any graph leads to a vertex coloring of its line graph, we obtain the following.

Corollary 7.8 1) If $n=2^{m}, m \geq 2$, then

$$
\chi\left(L\left(\overline{\Gamma\left(\mathbb{Z}_{n}[i]\right)}\right)\right)=2^{2 m-1}-3 .
$$

2) If $n=q^{m}, m \geq 3$, then

$$
\chi\left(L\left(\overline{\Gamma\left(\mathbb{Z}_{n}[i]\right)}\right)\right)=q^{2 m-2}-q^{2}-1 .
$$

3) If $n=p^{m}, m \geq 2$, then

$$
\chi\left(L\left(\overline{\Gamma\left(\mathbb{Z}_{n}[i]\right)}\right)\right)=2 p^{2 m-1}-p^{2 m-2}-p-1 .
$$

\section{The Domination Number of $\overline{\Gamma\left(\mathbb{Z}_{n}[i]\right)}$}

A subset $D$ of the vertex set $V(G)$ of a graph $G$ is a dominating set in $G$ if each vertex of $G$, not in $D$, is adjacent to at least one vertex of $D$. The minimum cardinality of all dominating sets in $G, \gamma(G)$, is called the domination number of $G$.

In $\overline{\Gamma\left(\mathbb{Z}_{2^{m}}[i]\right)}, m \geq 2$, the vertex $2^{m-1}+2^{m-1} i$ is an isolated vertex while the vertex $1+i$ dominates all vertices in the second component. Therefore, $\gamma\left(\overline{\Gamma\left(\mathbb{Z}_{2^{m}}[i]\right)}\right)=2$. The graph $\overline{\Gamma\left(\mathbb{Z}_{q^{2}}[i]\right)}=\left(q^{2}-1\right) K_{1}$, thus $\gamma\left(\overline{\Gamma\left(\mathbb{Z}_{q^{2}}[i]\right)}\right)=q^{2}-1$. In $\overline{\Gamma\left(\mathbb{Z}_{q^{m}}[i]\right)}, \quad m \geq 3$ the vertices $\alpha q^{m-1}+\beta q^{m-1} i$ are isolated while the vertex $q$ is adjacent to all other vertices in

$$
V\left(\overline{\Gamma\left(\mathbb{Z}_{q^{m}}[i]\right)}\right)-\left\{\alpha q^{m-1}+\beta q^{m-1} i: \alpha, \beta \in \mathbb{Z}_{q}\right\},
$$

so $\gamma\left(\overline{\Gamma\left(\mathbb{Z}_{q^{m}}[i]\right)}\right)=q^{2}$. Since

$$
\begin{gathered}
\Gamma\left(\mathbb{Z}_{q_{1} q_{2}}[i]\right)=K_{q_{1}^{2}-1} \cup K_{q_{2}^{2}-1} \\
\text { and } \overline{\Gamma\left(\mathbb{Z}_{p}[i]\right)}=K_{p-1} \cup K_{p-1}, \\
\gamma\left(\overline{\Gamma\left(\mathbb{Z}_{q_{1} q_{2}}[i]\right)}\right)=\gamma\left(\overline{\Gamma\left(\mathbb{Z}_{p}[i]\right)}\right)=2 .
\end{gathered}
$$

The set $D=\{(1,0),(0,1)\}$ is a minimum dominating set for $\overline{\Gamma\left(\mathbb{Z}_{p^{m}} \times \mathbb{Z}_{p^{m}}\right)}$. And if $n=n_{1} n_{2}$, where g.c.d $\left(n_{1}, n_{2}\right)=1$, then $\Gamma\left(\mathbb{Z}_{n}[i]\right) \cong \Gamma\left(\mathbb{Z}_{n_{1}}[i] \times \mathbb{Z}_{n_{2}}[i]\right)$. This graph is connected and the set $D=\{(1,0),(0,1)\}$ is a minimum dominating set for $\overline{\Gamma\left(\mathbb{Z}_{n_{1}}[i] \times \mathbb{Z}_{n_{2}}[i]\right)}$.

Theorem 8.1 1) If $n \neq 2, q^{m}$, then

$$
\gamma\left(\overline{\Gamma\left(\mathbb{Z}_{n}[i]\right)}\right)=2 \text {. }
$$

2) $\gamma\left(\overline{\Gamma\left(\mathbb{Z}_{q^{2}}[i]\right)}\right)=q^{2}-1$ and

$$
\gamma\left(\overline{\Gamma\left(\mathbb{Z}_{q^{m}}[i]\right)}\right)=q^{2}, m \geq 3 .
$$




\section{The Domination Number of $L\left(\overline{\Gamma\left(\mathbb{Z}_{n}[i]\right)}\right)$}

The independence number of $G, \beta(G)$, is the maximum cardinality of all independent sets in $G$. A subset $D$ of the edge set $V(G)$ of a graph $G$ is an edge dominating set in $G$ if each edge of $G$, not in $D$, is adjacent to at least one edge of $D$. The minimum cardinality of all edge dominating sets in $G, \gamma^{\prime}(G)$, is called the edge domination number of $G$. The minimum cardinality of all independent edge dominating sets, $\gamma_{i}^{\prime}(G)$, is called the independence edge domination number of $G$. The study of the domination number of the line graph of $G$ leads to the study of edge or line domination number of $G$, i.e. $\gamma(L(G))=\gamma^{\prime}(G)$. On the other hand, for any graph $G, \gamma_{i}^{\prime}(G)=\gamma^{\prime}(G)$ [15].

If $S$ is an independent set in $G$, then $S$ induces a complete graph in $G$. While if $S$ induces a complete graph in $G$, then it is independent in $G$. Recall that $\mathbb{Z}_{{ }^{m}}[i] \cong \mathbb{Z}_{2^{2 m}} \quad[2]$. Then the sets,

$A_{j}=\left\{\alpha 2^{j}: \alpha \in U\left(\mathbb{Z}_{2^{2 m-j}}\right)\right\}, \quad j=1,2, \cdots, 2 m-1$ form a partition for the set $V\left(\Gamma\left(\mathbb{Z}_{2^{2 m}}\right)\right)$. Clearly, the set $T=\bigcup_{j=m}^{2 m-1} A_{j}$ is the maximum independent set in

$\overline{\Gamma\left(\mathbb{Z}_{2^{2 m}}\right)}$, while the set $S=\bigcup_{j=1}^{m-1} A_{j}$ induces a maximum complete subgraph in $\overline{\Gamma\left(\mathbb{Z}_{2^{2 m}}\right)}$. There are some edges joining $S$ to $T$, no other adjacency exists in $\overline{\Gamma\left(\mathbb{Z}_{2^{2 m}}\right)}$. Any edge dominating set for $\overline{\Gamma\left(\mathbb{Z}_{2^{2 m}}\right)}$ must contain at least $[|S| / 2\rceil$ element in order to dominate $\langle S\rangle$. On the other hand, this dominating set for $\langle S\rangle$ dominates all other edges in $\overline{\Gamma\left(\mathbb{Z}_{2^{2 m}}\right)}$. Since

$\left|A_{j}\right|=2^{2 m-j-1}$, then $|S|$ and $|T|$, could easily be computed to get the following theorem.

Theorem 9.1 For $n=2^{m}, m \geq 2$.

1) $\omega\left(\overline{\Gamma\left(\mathbb{Z}_{n}[i]\right)}\right)=2^{m}\left(2^{m-1}-1\right)$.

2) $\beta\left(\overline{\Gamma\left(\mathbb{Z}_{n}[i]\right)}\right)=2^{m}-1$.

3) $\gamma\left(L\left(\overline{\Gamma\left(\mathbb{Z}_{n}[i]\right)}\right)\right)=\gamma_{i}^{\prime}\left(\overline{\Gamma\left(\mathbb{Z}_{n}[i]\right)}\right)$ $=\gamma^{\prime}\left(\overline{\Gamma\left(\mathbb{Z}_{n}[i]\right)}\right)=2^{m-1}\left(2^{m-1}-1\right)$.

To study the graph $\overline{\Gamma\left(\mathbb{Z}_{q^{m}}[i]\right)}, m \geq 3$, consider the partition of $\overline{\Gamma\left(\mathbb{Z}_{q^{m}}[i]\right)}$ given by

$$
\begin{aligned}
& A_{k j}=\left\{\alpha q^{k}+\beta q^{j} i: \alpha \in U\left(\mathbb{Z}_{q^{m-k}}\right) \text { and } \beta \in U\left(\mathbb{Z}_{q^{m-j}}\right)\right\}, \\
& 1 \leq k, j \leq m .
\end{aligned}
$$

and not both $j, k=m$. The set $T=\left(\bigcup_{k=\left\lceil\frac{m}{2}\right\rceil}^{m}\left(\bigcup_{j=\left\lceil\frac{m}{2}\right\rceil}^{m} A_{k j}\right)\right)-A_{m m}$ is the maximum independent set, while $S=\bigcup_{j=1}^{\left[\frac{m}{2}\right]-1}\left(\bigcup_{k=1}^{\left[\frac{m}{2}\right]-1} A_{k j}\right)$ induces a maximum complete subgraph in $\overline{\Gamma\left(\mathbb{Z}_{q^{m}}[i]\right)}$. There are some edges joining $S$ to $T$, and $\overline{\Gamma\left(\mathbb{Z}_{q^{m}}[i]\right)}$ has no other adjacency. Easy calculations give $\mid \begin{aligned} & A_{k j} \mid=(q-1)^{2} q^{2 m-k-j-2} \text { when } 1 \leq k, j \leq m-1, \\ & A_{m j} \mid=q^{m-j}-q^{m-j-1} \text { and }\left|A_{k m}\right|=q^{m-k}-q^{m-k-1} \text { when }\end{aligned}$ $k, j \neq m$. While $|T|=q^{2\left\lfloor\frac{m}{2}\right\rfloor}-1$ and $|S|=q^{2\left\lfloor\frac{m}{2}\right]}\left(q^{\left[\frac{m}{2}\right]}-1\right)^{2}$.

Thus we obtain the following theorem.

Theorem 9.2 If $n=q^{m}, m \geq 3$, then
1) $\omega\left(\overline{\Gamma\left(\mathbb{Z}_{n}[i]\right)}\right)=q^{2\left\lfloor\frac{m}{2}\right\rfloor}\left(q^{\left[\frac{m}{2}\right]}-1\right)^{2}$.
2) $\beta \overline{\left(\Gamma\left(\mathbb{Z}_{n}[i]\right)\right)}=q^{m}-1$ if $m$ is even and $q^{m-1}$ if $m$ is odd.

$$
\begin{aligned}
& \gamma\left(L\left(\left(\overline{\Gamma\left(\mathbb{Z}_{n}[i]\right)}\right)\right)\right)=\gamma_{i}^{\prime}\left(\left(\overline{\Gamma\left(\mathbb{Z}_{n}[i]\right)}\right)\right) \\
& =\gamma^{\prime}\left(\left(\overline{\Gamma\left(\mathbb{Z}_{n}[i]\right)}\right)\right)=\frac{1}{2} q^{2\left[\frac{m}{2}\right]}\left(q^{\left[\frac{m}{2}\right]}-1\right)^{2} .
\end{aligned}
$$

3)

Now, we move to the case $n=p^{m}$. Let

$$
A_{k j}=\left\{\left(\alpha p^{k}, \beta p^{j}\right): \alpha \in U\left(\mathbb{Z}_{p^{m-k}}\right) \text { and } \beta \in U\left(\mathbb{Z}_{p^{m-j}}\right)\right\} \text {. }
$$

Clearly, the sets $A_{k j}$ where $0 \leq k, j \leq m$ and not both $k, j=m$ or 0 , partition the vertices of

$$
\begin{aligned}
\overline{\Gamma\left(\mathbb{Z}_{p^{m}} \times \mathbb{Z}_{p^{m}}\right)} \text { and }\left|A_{k j}\right|=p^{2 m-k-j-2}(p-1)^{2} . \text { Let } \\
S_{1}=\left(\bigcup_{k=1}^{m} A_{k 0}\right) \cup\left(\bigcup_{k=1}^{m-1} A_{0 k}\right) \\
S_{2}=\bigcup_{k=1}^{\left\lceil\frac{m}{2}\right\rceil-1}\left(\bigcup_{j=1}^{\frac{m}{2} \mid-1} A_{k j}\right), S_{3}=\bigcup_{k=\left\lceil\frac{m}{2}\right]}^{m}\left(\bigcup_{\left.j=\mid \frac{m}{2}\right\rceil}^{m} A_{k j}\right), \\
\left.S_{4}=\bigcup_{j=1}^{\left\lceil\frac{m}{2}\right\rceil-1}\left(\bigcup_{k=\left\lceil\frac{m}{2}\right\rceil}^{m} A_{k j}\right) \text { and } S_{5}=\bigcup_{k=1}^{\left\lceil\frac{m}{2} \mid-1\right.} \bigcup_{\left.j=\mid \frac{m}{2}\right\rceil}^{m} A_{k j}\right) .
\end{aligned}
$$

Note that $S_{1}$ induces a complete graph in 
$\overline{\Gamma\left(\mathbb{Z}_{p^{m}} \times \mathbb{Z}_{p^{m}}\right)}$. Vertices in $\bigcup_{k=1}^{m-1} A_{k 0}$ are adjacent to all vertices except some vertices in $\bigcup_{k=1}^{m-1} A_{k m}$. Similarly, vertices in $\bigcup_{k=1}^{m-1} A_{0 k}$ are adjacent to all vertices except some vertices in $\bigcup_{k=1}^{m-1} A_{m k}$, and vertices in $A_{m 0}$ are adjacent to all vertices except vertices in $A_{0 m}$. On the other hand $A_{0 m}$ induces a complete subgraph and vertices in this set are adjacent to all other vertices except those of $A_{m 0}$. Clearly $S_{2}$ induces a complete subgraph. Vertices in $S_{3}$ form an independent set, and are adjacent to some vertices in $S_{1} \cup S_{2} \cup S_{4} \cup S_{5} \cup A_{0 m}$. Each of $S_{4}$ and $S_{5}$ induces a complete subgraph and are adjacent to some vertices in $S_{1} \cup S_{2} \cup S_{3} \cup A_{0 m}$. Besides, there are some edges between $S_{4}$ and $S_{5}$. On the other hand,

$$
\left|S_{3}\right|=\sum_{\left.k=\frac{m}{2}\right] j=\left\lceil\frac{m}{2}\right\rceil}^{m} \sum_{k j}^{m}|-| A_{m m} \mid .
$$

The above argument shows that

$$
\begin{aligned}
& \gamma\left(L\left(\overline{\Gamma\left(\mathbb{Z}_{p^{m}}[i]\right)}\right)\right)=\gamma_{i}^{\prime}\left(\overline{\Gamma\left(\mathbb{Z}_{p^{m}}[i]\right)}\right) \\
& =\gamma^{\prime}\left(\overline{\Gamma\left(\mathbb{Z}_{p^{m}}[i]\right)}\right)=\frac{1}{2}\left(\left|\overline{\Gamma\left(\mathbb{Z}_{p^{m}}[i]\right)}\right|-\left|S_{3}\right|\right) \\
& =\frac{1}{2}\left(2 P^{2 m-1}-p^{2 m-2}-p^{2 m-\left|\frac{m}{2}\right|}-2\right) .
\end{aligned}
$$

\section{The Degree of the Vertices in $\Gamma\left(\mathbb{Z}_{n}[i]\right)$ and $L\left(\Gamma\left(\mathbb{Z}_{n}[i]\right)\right)$}

Now, we determine the cardinality of the annihilator of the element $a+b i$, ann $(a+b i)$ in $\mathbb{Z}_{n}[i]$. This helps find the degree of each vertex in $\Gamma\left(\mathbb{Z}_{n}[i]\right)$, its complement, as well as the degree of each vertex in their corresponding line graphs.

Theorem 10.1 If $a+b i \in \mathbb{Z}_{n}[i]$, then $|a n n(a+b i)|=c^{2}+d^{2}$ where g.c.d $(a+b i, n)=c+d i$.

Proof. Let $a+b i \in \mathbb{Z}_{n}[i]$ and g.c.d $(a+b i, n)=c+d i$. Then $a n n(a+b i)=\{x \in \mathbb{Z}[i]: x(a+b i) \equiv 0(\bmod n)\}$.

So, $x .(a+b i) \equiv 0(\bmod n) \Leftrightarrow x \cdot \frac{a+b i}{c+d i} \equiv 0\left(\bmod \frac{n}{c+d i}\right)$.

$$
\text { But } \frac{a+b i}{c+d i} \in U\left(\mathbb{Z}_{\frac{n}{c+d i}}[i]\right) \text {. So, } x \equiv 0\left(\bmod \frac{n}{c+d i}\right)
$$

and hence there exists $m \in \mathbb{Z}[i]$ such that $x=\frac{n}{c+d i} m$.

Since $m=t(c+d i)+r$ where $t, r \in \mathbb{Z}[i]$ and the norm of $r$ is less than the norm of $c+d i$,

$|\operatorname{ann}(a+b i)|=\left|\left\{r: r \in \mathbb{Z}_{c+d i}[i]\right\}\right|=\left|\mathbb{Z}_{c+d i}[i]\right|$. By Theorem 2 of [7], $\left|\mathbb{Z}_{c+d i}[i]\right|=\left|\mathbb{Z}_{c^{2}+d^{2}}\right|=c^{2}+d^{2}$, so the result holds.

Theorem 10.2 Let $v \in V\left(\Gamma\left(\mathbb{Z}_{n}[i]\right)\right)$ and g.c.d $(v, n)=c+d i$. Then

$$
\operatorname{deg}(v)=\left\{\begin{array}{ll}
c^{2}+d^{2}-1, & \text { if } v^{2} \neq 0 \\
c^{2}+d^{2}-2, & \text { if } v^{2}=0
\end{array} .\right.
$$

The order of $\Gamma\left(\mathbb{Z}_{n}[i]\right)$ can be easily computed using formulas given in [1]. Thus we can find the degree of each vertex in the complement of $\Gamma\left(\mathbb{Z}_{n}[i]\right)$, here we give the degree of each vertex in the line graph of $\Gamma\left(\mathbb{Z}_{n}[i]\right)$, an analogous formula for the degree of vertices in $L\left(\Gamma\left(\mathbb{Z}_{n}[i]\right)\right)$ could be obtained.

Corollary 10.3 Let $[u, v] \in V\left(L\left(\Gamma\left(\mathbb{Z}_{n}[i]\right)\right)\right)$, g.c.d $(u, n)=a+b i$ and g.c.d $(v, n)=c+d i$. Then

$$
\begin{aligned}
& \operatorname{deg}([u, v]) \\
& =\left\{\begin{array}{ll}
a^{2}+b^{2}+c^{2}+d^{2}-4, & \text { if } u^{2} \neq 0 \text { and } v^{2} \neq 0 \\
a^{2}+b^{2}+c^{2}+d^{2}-5, & \text { if } u^{2}=0 \text { and } v^{2} \neq 0 \\
a^{2}+b^{2}+c^{2}+d^{2}-6, & \text { if } u^{2}=0 \text { and } v^{2}=0
\end{array} .\right.
\end{aligned}
$$

Proof. Note that, for any graph $G$ and $u v \in E(G)$, $\operatorname{deg}_{L(G)}([u, v])=\operatorname{deg}_{G}(u)+\operatorname{deg}_{G}(v)-2$.

In the following we determine the degree of every vertex in the graphs $\Gamma\left(\mathbb{Z}_{n}[i]\right)$ when $n=2^{m}, m \geq 2, n=q^{m}, m \geq 3$ and $n=p^{m}, m \geq 1$.

Theorem 10.4 Let $n=2^{m}, m \geq 3$ and $\alpha, \beta$ are odd. Then in $\Gamma\left(\mathbb{Z}_{n}[i]\right)$,

1) $\operatorname{deg}\left(\alpha 2^{k}+\beta 2^{s} i\right)=\left\{\begin{array}{ll}2^{2 k}-1, & \text { if } 1 \leq k<s<m \text { and } k<\left\lceil\frac{m}{2}\right\rceil \text { or } 1 \leq k=s<\left\lfloor\frac{m}{2}\right\rfloor \text { and } \alpha \neq \pm \beta \\ 2^{2 k}-2, & \text { if }\left\lceil\frac{m}{2}\right\rfloor \leq k<s<m \text { or }\left\lfloor\frac{m}{2}\right\rfloor \leq k=s<m \text { and } \alpha \neq \pm \beta \\ 2^{2 k+1}-2, & \text { if }\left\lfloor\frac{m}{2}\right\rfloor \leq k=s<m \text { and } \alpha= \pm \beta \\ 2^{2 k+1}-1, & \text { if } 1 \leq k=s<\left\lfloor\frac{m}{2}\right\rfloor \text { and } \alpha= \pm \beta\end{array}\right.$. 
2) $\operatorname{deg}\left(\alpha 2^{k}\right)=\operatorname{deg}\left(\beta 2^{k} i\right)$

$$
=\left\{\begin{array}{ll}
2^{2 k}-1, & \text { if } 1 \leq k<\left\lceil\frac{m}{2}\right\rceil \\
2^{2 k}-2, & \text { if }\left\lceil\frac{m}{2}\right\rceil \leq k<m
\end{array} .\right.
$$

3) $\operatorname{deg}(\alpha+\beta i)=1$.

Proof. 1) Note that, g.c.d $\left(n, \alpha 2^{k}+\beta 2^{s} i\right)=2^{\min \{k, s\}}$ if $k \neq s$ or $\alpha \neq \beta$ and g.c.d $\left(n, \alpha 2^{k}+\beta 2^{s} i\right)=2^{k}(1 \pm i)$ if and only if $k=s$ and $\alpha= \pm \beta$. Moreover $\left(\alpha 2^{k}+\beta 2^{k} i\right)^{2}=0$ if and only if $k \geq\left\lfloor\frac{m}{2}\right\rfloor$.

2) Obvious.

3) Note that if $\alpha, \beta$ are odd, then g.c.d $(\alpha+\beta i, n)=1+i$.

Theorem 10.5 Let $n=q^{m}, m \geq 3, \quad \alpha, \beta$ are relatively prime with $q$. Then in $\Gamma\left(\mathbb{Z}_{n}[i]\right)$,

$$
\operatorname{deg}\left(\alpha q^{k}+\beta q^{s} i\right)=\left\{\begin{array}{ll}
q^{2 k}-1, & \text { if } 1 \leq k \leq s \text { and } k<\left\lceil\frac{m}{2}\right\rceil \\
q^{2 k}-2, & \text { if }\left\lceil\frac{m}{2}\right\rceil \leq k \leq s
\end{array} .\right.
$$

Theorem 10.6 Let $n=p^{m}, m \geq 1, \quad p=a^{2}+b^{2}$ and g.c.d $(\alpha, p)=1$. Then in $\Gamma\left(\mathbb{Z}_{n}[i]\right)$,

$$
\begin{aligned}
& \operatorname{deg}\left(\alpha(a+b i)^{k}(a-b i)^{s}\right) \\
& = \begin{cases}\left(a^{2}+b^{2}\right)^{k+s}-1, & \text { if } k \text { or } s<\left\lceil\frac{m}{2}\right\rceil \text { and } k, s \geq 1 \\
\left(a^{2}+b^{2}\right)^{k+s}-2, & \text { if } k, s \geq\left\lceil\frac{m}{2}\right\rceil \\
\left(a^{2}+b^{2}\right)^{s}-1, & \text { if } k=0 \\
\left(a^{2}+b^{2}\right)^{k}-1, & \text { if } s=0\end{cases}
\end{aligned}
$$

\section{When Is $L\left(\Gamma\left(\mathbb{Z}_{n}[i]\right)\right), L\left(\overline{\Gamma\left(\mathbb{Z}_{n}[i]\right)}\right)$ \\ Regular?}

A graph $G$ in which all vertices have the same degree is called regular graph.

Regularity of $\Gamma\left(\mathbb{Z}_{n}[i]\right)$ was studied in [1]. However, we provide our own proof, since it comes as an immediate consequence of Theorem 10.2. Clearly, if $n=2, p, q^{2}$, then $\Gamma\left(\mathbb{Z}_{n}[i]\right)$ is regular. If

$n=2^{m}, m \geq 2$ or $n=q^{m}, m \geq 3$, then the graph $\Gamma\left(\mathbb{Z}_{n}[i]\right)$ has a vertex which is adjacent to all other vertices and it is not complete graph, thus $\Gamma\left(\mathbb{Z}_{n}[i]\right)$ is not regular.

Now, we show that $\Gamma\left(\mathbb{Z}_{n}[i]\right)$ is regular if and only if $n=2, p, q^{2}$.

Theorem 11.1 If $n=\prod_{j=1}^{r} \pi_{j}^{m_{j}}$ where $\pi_{j}^{\prime} s$ are distinct Gaussian primes and $m_{j} \geq 1$ and $n \neq 2^{m}, p^{m}, q^{m}, m \geq 2$, then $\Gamma\left(\mathbb{Z}_{n}[i]\right)$ is not regular.

Proof. Choose two vertices $\pi_{t}$ and $\pi_{s}$ such that $\pi_{t} \neq \overline{\pi_{s}}$, then $\operatorname{g.c.d}\left(n, \pi_{t}\right)=\pi_{t} \neq \operatorname{g.c.d}\left(n, \pi_{s}\right)=\pi_{s}$. So, the result follows.

Next, we discuss regularity of the graph $L\left(\Gamma\left(\mathbb{Z}_{n}[i]\right)\right)$ and $L\left(\overline{\Gamma\left(\mathbb{Z}_{n}[i]\right)}\right)$. Clearly, if $G$ is regular, then $L(G)$ is also regular, so if $n=p, q^{2}$, then the graph $L\left(\Gamma\left(\mathbb{Z}_{n}[i]\right)\right)$ is regular. On the other hand, if $G$ is the complete bipartite graph $K_{r, s}$, then $\operatorname{deg}([u, v])=r+s-2$ for all vertices in $L\left(K_{r, s}\right)$. Thus $L\left(\Gamma\left(\mathbb{Z}_{q_{1} q_{2}}[i]\right)\right)$ is regular. While $\Gamma\left(\mathbb{Z}_{2}[i] \times \mathbb{Z}_{q}[i]\right)$ is a bipartite graph with partite sets

$$
\begin{aligned}
A= & \{(1+i, 0),(1,0),(i, 0)\} \text { and } \\
B= & \left\{(1+i, x): x \in V\left(\Gamma\left(\mathbb{Z}_{q}[i]\right)\right)\right\} \\
& \cup\left\{(0, x): x \in V\left(\Gamma\left(\mathbb{Z}_{q}[i]\right)\right)\right\} .
\end{aligned}
$$

Moreover, $N((1+i, 0))=B, N((1+i, 1))=\{(1+i, 0)\}$ and $N((0,1))=A$. Thus,

$$
\operatorname{deg}([(1+i, 0),(1+i, 1)]) \neq \operatorname{deg}([(1+i, 0),(0,1)]),
$$

and hence, $L\left(\Gamma\left(\mathbb{Z}_{2 q}[\mathbf{i}]\right)\right)$ is not regular.

Theorem 11.2 If $n=t^{m}, m \geq 2, t$ is a prime and $n \neq q^{2}$, then the graph $L\left(\Gamma\left(\mathbb{Z}_{n}[i]\right)\right)$ is not regular.

Proof. If $n=2^{m}, m \geq 2$, then $\operatorname{deg}\left(\left[1+i, 2^{m-1}+2^{m-1} i\right]\right) \neq \operatorname{deg}\left(\left[2,2^{m-1} i\right]\right)$. If $n=q^{m}, m \geq 3$, then $\operatorname{deg}\left(\left[q, q^{m-1} i\right]\right) \neq \operatorname{deg}\left(\left[q^{2}, q^{m-1} i\right]\right)$. And if $n=p^{m}, \quad p=a^{2}+b^{2}, \quad m \geq 2$, then

$$
\begin{aligned}
& \operatorname{deg}\left(\left[(a+b i)^{m},(a-b i)^{m}\right]\right) \\
& \neq \operatorname{deg}\left[(a+b i),(a-b i)^{m}(a+b i)^{m-1}\right] .
\end{aligned}
$$

Theorem 11.3 Let $R=R_{1} \times R_{2}$ where $R_{1}$ and $R_{2}$ are commutative rings with unity with at least one of them is not ID. Then $L(\Gamma(R))$ is not regular.

Proof. Suppose that $R_{1}$ is not ID and $\left|R_{i}\right|=r_{i}$, for $i=1,2$. Let $x_{1} \in V\left(\Gamma\left(R_{1}\right)\right)$. If $x_{1}^{2}=0$, then

$$
\begin{aligned}
N\left(\left(x_{1}, 0\right)\right) & =\left\{(0, a): a \in R_{2}-\{0\}\right\} \\
& \cup\left\{(y, a): y \in \operatorname{ann}\left(x_{1}\right)-\left\{0, x_{1}\right\}\right.
\end{aligned}
$$

and $a \in R_{2}$ if

$\left\{\operatorname{ann}\left(x_{1}\right)-\left\{0, x_{1}\right\} \neq \varphi\right\} \cup\left\{\left(x_{1}, a\right): a \in R_{2}-\{0\}\right\}$, hence

$\operatorname{deg}\left(\left[\left(x_{1}, 0\right),(0,1)\right]\right) \geq 2 r_{2}+r_{1}-4$. And if $x_{1}^{2} \neq 0$, 


$$
\begin{aligned}
\left(N\left(x_{1}, 0\right)\right) & =\left\{(0, a): a \in R_{2}-\{0\}\right\} \\
& \cup\left\{(y, a): y \in \operatorname{ann}\left(x_{1}\right)-\left\{0, x_{1}\right\}\right\}
\end{aligned}
$$

and $a \in R_{2}$ if $\left\{\operatorname{ann}\left(x_{1}\right)-\left\{0, x_{1}\right\} \neq \varphi\right.$, hence

$\left.\operatorname{deg}\left(\left[\left(x_{1}, 0\right),(0,1)\right]\right) \geq r_{2}+r_{1}-3\right\}$. But

$\operatorname{deg}[(1,0),(0,1)]=r_{1}+r_{2}-4$. So $L(\Gamma(R))$ is not regular.

So as a consequence of Theorem 11.2 and Theorem 11.3 , we conclude the following.

Theorem 11.4 The graph $L\left(\Gamma\left(\mathbb{Z}_{n}[i]\right)\right)$ is regular if and only if $n=p, q^{2}, q_{1} q_{2}$.

Observe that, for $n=2, q^{2}, \Gamma\left(\mathbb{Z}_{n}[i]\right)$ is the empty graph. $\overline{\Gamma\left(\mathbb{Z}_{q^{3}}[i]\right)}=N_{q^{2}-1} \cup K_{q^{4}-q^{2}}$, so the line graph

$L\left(\overline{\Gamma\left(\mathbb{Z}_{q^{3}}[i]\right)}\right)$ is regular. While

$\overline{\Gamma\left(\mathbb{Z}_{n}[i]\right)}=K_{p-1} \cup K_{p-1}$

which is regular, so is $L\left(\overline{\Gamma\left(\mathbb{Z}_{n}[i]\right)}\right)$.

$$
\begin{gathered}
\operatorname{In} L\left(\overline{\Gamma\left(\mathbb{Z}_{2^{m}}[i]\right)}\right), \operatorname{deg}([1+i, 1+3 i]) \neq \operatorname{deg}[1+i, 2] \\
\operatorname{In}\left(\overline{\Gamma\left(\mathbb{Z}_{q^{m}}[i]\right)}\right), m>3, \operatorname{deg}[q, q i] \neq \operatorname{deg}\left[q^{2}, q\right] .
\end{gathered}
$$

And in $L\left(\overline{\Gamma\left(\mathbb{Z}_{p^{m}}[i]\right)}\right), m \geq 2$,

$\operatorname{deg}[a+b i, a-b i] \neq \operatorname{deg}\left[(a+b i)^{2}, a-b i\right]$. So, the graph $L\left(\overline{\Gamma\left(\mathbb{Z}_{n}[i]\right)}\right)$ is not regular for $n=t^{m}, m \geq 2, t$ is a prime and $n \neq q^{2}, q^{3}$.

Theorem 11.5 Let $R=R_{1} \times R_{2}$ where $R_{1}$ and $R_{2}$ are commutative rings with unity such that $|V(\Gamma(R))|=t, \quad\left|R_{i}\right|=r_{i}$ for $i=1,2$. If $\left|\operatorname{reg}\left(R_{i}\right)\right| \geq 2$ and $r_{1} \neq r_{2}$, then $L \overline{(\Gamma(R))}$ is not regular.

Proof. Since $\left|\operatorname{reg}\left(R_{i}\right)\right| \geq 2$, for $i=1,2$, there exist $u_{1} \in \operatorname{reg}\left(R_{1}\right)-\{1\}$ and $u_{2} \in \operatorname{reg}\left(R_{2}\right)-\{1\}$. Therefore $\left[(1,0),\left(u_{1}, 0\right)\right],\left[(0,1),\left(0, u_{2}\right)\right] \in V(L(\overline{\Gamma(R)}))$. Since

$$
\begin{gathered}
r_{1} \neq r_{2}, \\
\operatorname{deg}\left(\left[(1,0),\left(u_{1}, 0\right)\right]\right)=2 t-2 r_{2}-4 \neq 2 t-2 r_{1}-4 \\
=\operatorname{deg}\left(\left[(0,1),\left(0, u_{2}\right)\right]\right)
\end{gathered}
$$

So, $L(\overline{\Gamma(R)})$ is not regular.

Theorem 11.6 The graph $L \overline{\left(\Gamma\left(\mathbb{Z}_{n}[i]\right)\right)}$ is regular if and only if $n=p$ or $q^{3}$.

\section{When is $L\left(\Gamma\left(\mathbb{Z}_{n}[i]\right)\right), \quad L\left(\overline{\Gamma\left(\mathbb{Z}_{n}[i]\right)}\right)$}

\section{Locally $H$ ?}

A simple graph $G$ is said to be locally $H$ if the neighborhood of each vertex in $V(G)$ induces the same graph $H$. The cartesian product $G \square H$ of two graphs $G$ and $H$ is the graph with vertex set $V(G \square H)=V(G) \times V(H)$ and two vertices in $V(G \square H)$ are adjacent if and only if they are equal in one coordinate and adjacent in the other. Before we proceed, we give the following lemma.

Lemma 12.1 1) If $G=K_{n}, n \geq 3$, then $L(G)$ is locally $K_{n-2} \square K_{2}$.

2) If $G=K_{m, n}, m, n \geq 2$, then $L(G)$ is locally $K_{m-1} \cup K_{n-1}$.

Proof. 1) Let $[u, v] \in V\left(L\left(K_{n}\right)\right)$, then

$$
\begin{aligned}
N([u, v])= & \left\{[u, a]: a \in V\left(K_{n}\right)-\{u, v\}\right\} \\
& \cup\left\{[a, v]: a \in V\left(K_{n}\right)-\{u, v\}\right\}
\end{aligned}
$$

each of the sets $\left\{[u, a]: a \in V\left(K_{n}\right)-\{u, v\}\right\}$ and $\left\{[a, v]: a \in V\left(K_{n}\right)-\{u, v\}\right\}$ induces a copy of $K_{n-2}$ and since we deal with an undirected graphs, then for a fixed $a,[u, a]$ and $[v, a]$ are adjacent. Thus the result holds.

3) Let $[u, v] \in V\left(L\left(K_{m, n}\right)\right)$, with partite sets $A$ and $B$ and with $u \in A, v \in B$. Then

$$
\begin{aligned}
N([u, v])= & \{[u, b]: b \in B-\{v\}\}\} \\
& \cup\{[a, v]: a \in A-\{u\}\}
\end{aligned} .
$$

Each set induces a complete graph $K_{n-1}, K_{m-1}$, respectively. And $\langle N([u, v])\rangle$ has no other edges. Thus $N([u, v])$ induces $K_{n-1} \cup K_{m-1}$.

In order for a graph to be locally $H$, it should be regular graph. Thus for the graph $L\left(\Gamma\left(\mathbb{Z}_{n}[i]\right)\right)$, it suffices to check the cases $n=p, q^{2}, q_{1} q_{2}$, and for $L\left(\overline{\Gamma\left(\mathbb{Z}_{n}[i]\right)}\right)$, we consider only the cases $n=p, q^{3}$. Since $\Gamma\left(\mathbb{Z}_{P}[i]\right)=K_{p-1, p-1}$ and $\overline{\Gamma\left(\mathbb{Z}_{P}[i]\right)}=K_{p-1} \cup K_{p-1}$, $L\left(\Gamma\left(\mathbb{Z}_{P}[i]\right)\right)$ is locally $K_{p-2} \cup K_{p-2}$ and $L\left(\overline{\Gamma\left(\mathbb{Z}_{P}[i]\right)}\right)$ is locally $K_{p-2} \square K_{2}$. In the same manner we can show that $L\left(\Gamma\left(\mathbb{Z}_{q_{1} q_{2}}[i]\right)\right)$ is locally $K_{q_{1}^{2}-2} \cup K_{q_{2}^{2}-2}, L\left(\Gamma\left(\mathbb{Z}_{q^{2}}[i]\right)\right)$ is locally $K_{q^{2}-2} \square K_{2}$ and $L\left(\overline{\Gamma\left(\mathbb{Z}_{q^{3}}[i]\right)}\right)$ is locally $K_{q^{4}-q^{2}-2} \square K_{2}$.

Theorem 12.2 The following statements are equivalent.

1) The graph $L\left(\Gamma\left(\mathbb{Z}_{n}[i]\right)\right) / L\left(\overline{\Gamma\left(\mathbb{Z}_{n}[i]\right)}\right)$ is regular, 
2) The graph $L\left(\Gamma\left(\mathbb{Z}_{n}[i]\right)\right) / L\left(\overline{\Gamma\left(\mathbb{Z}_{n}[i]\right)}\right)$ is locally $H$.

\section{REFERENCES}

[1] E. Abu Osba, S. Al-Addasi and N. Abu Jaradeh, "Zero Divisor Graph for the Ring of Gaussian Integers Modulo n," Communication in Algebra, Vol. 36, No. 10, 2008, pp. 3865-3877. doi:10.1080/00927870802160859

[2] E. Abu Osba, S. Al-Addasi and B. Al-Khamaiseh, "Some Properties of the Zero Divisor Graph for the Ring of Gaussian Integers Modulo n," Glasgow Journal of Math, Vol. 53, No. 1, 2011, pp. 391-399. doi:10.1017/S0017089511000024

[3] E. Abu Osba, "The Complement Graph for Gaussian Integers Modulo n," Communication in Algebra, accepted.

[4] K. Nazzal and M. Ghanem, "On the Line Graph of the Zero Divisor Graph for the Ring of Gaussian Integers Modulo n," International Journal of Combinatorics, Vol. 2012, Article ID 957284. doi:10.1155/2012/957284

[5] P. F. Lee, "Line Graph of Zero Divisor Graph in Commutative Rings," Master's Thesis, Colorado Christian University, 2007.

[6] J. Sedlàcek, "Some Properties of Interchange Graphs, Theory of Graphs and Its Applications," Academic Press, New York, 1962, pp. 145-150.

[7] G. Dersden and W. M. Dymcek, "Finding Factors of Factor Rings over the Gaussian Integers," American
Mathematical Monthly, Vol. 112, No. 7, 2005, pp. 602 611. doi: $10.2307 / 30037545$

[8] V. G. Vising, "The Number of Edges in a Graph of a Given Radius," Soviet Mathematics-Doklady, Vol. 8, 1967, pp. 535-536.

[9] C. Berg, "Graphs and Hypergraphs," American Elsevier Publishing Co, Inc., New York, 1976.

[10] H. J. Veldman, "A Result on Hamiltonian Line Graphs Involving Restrictions on Induced Subgraphs," Journal of Graph Theory, Vol. 12, No. 3, 1988, pp. 413-420. doi:10.1002/igt.3190120312

[11] D. J. Oberly and D. P. Sumner, "Every Connected, Locally Connected Nontrivial Graph with No Induced Claw Is Hamiltonian," Journal Graph Theory, Vol. 3, No. 4, 1979, pp. 351-356. doi:10.1002/jgt.3190030405

[12] M. J. Plantholt, "The Chromatic Index of Graphs with Large Maximum Degree," Discrete Mathematics, Vol. 47, 1983, pp.91-96. doi:10.1016/0012-365X(83)90074-2

[13] B.-L. Chen and H.-L. Fu, "Total Chromatic Number and Chromatic Index of Split Graphs," The Journal of Combinatorial Mathematics and Combinatorial Computing, Vol. 17, 1995, pp. 137-146.

[14] S. Akbari and A. Mohamamadaian, "On the Zero Divisor Graph of a Commutative Ring," Journal of Algebra, Vol. 274, No. 2, 2004, pp. 847-855. doi:10.1016/S0021-8693(03)00435-6

[15] S. Arumugam and S. Velammal, "Edge Domination in Graphs," Taiwanese Journal of Mathematics, Vol. 2, No. 2, 1998, pp. 173-179. 